PACS 73.61.At, 36.40.Vz, 68.55.Ln, 68.65.Cd 71.15.Mb, 73.30. $+\mathrm{y}$, $32.10 . \mathrm{Hq}$

\section{SIZE DEPENDENCES OF THE ENERGY PARAMETERS OF CHARGED METAL CLUSTERS WITH A MONOVACANCY}

\begin{abstract}
Radial distributions of electron concentration and electrostatic potential in perfect clusters and clusters with a centered monovacancy have been calculated self-consistently in the framework of the stabilized jellium model and using the Kohn-Sham method, which allowed the total energy of neutral and charged clusters containing a defect to be determined for the first time. On the basis of the results obtained, the dissociation, cohesive, and monovacancy formation energies, the electron affinity, the ionization potential, and the electric capacitance are directly calculated. The results of numerical calculations for $\mathrm{Na}, \mathrm{Mg}$, and $\mathrm{Al}$ are compared with asymptotic dependences and with the results obtained for defect-free clusters. The quantumsize dependences of the energy of monovacancy formation driven by either the Schottky or the "bubble blowing" mechanism, as well as their asymptotics, are determined. Strong fluctuations of this quantity as a function of the cluster size are revealed. The asymptotic dependences obtained for two indicated mechanisms are shown to differ from each other, but depend weakly on the number of atoms in a cluster.
\end{abstract}

Ke ywords: metal clusters, cohesion, dissociation, energy of vacancy formation, work function, ionization potential, electron affinity.

\section{Introduction}

One of the models describing the melting of solids [1] predicts that the concentration of vacancies should increase in a jump-like manner at the triple point, and the energy of their formation should decrease, as the vacancy concentration grows. At the melting point, the concentration of vacancies in metals amounts to a few tenths of percent. Despite such low concentrations, vacancies substantially affect the properties of solids.

In order to estimate the equilibrium concentration of vacancies, one has to know the vacancy formation energy. The magnitude of this parameter can be obtained by analyzing the annihilation spectrum of

(c) V.V. POGOSOV, V.I. REVA, 2017 positrons injected into a metal. It was found experimentally that the melting temperature of free clusters and clusters on a substrate decreases as their size diminishes. A significant number of works were devoted to the interpretation and the modeling of this mesoscopic phenomenon [2-7].

Modern mass spectrometric and calorimetric methods make it possible to comprehensively study the processes of premelting and melting in metal clusters consisting of a denumerable number of atoms [8-11]. In works $[8,10]$, it was shown that the melting temperature, besides size-induced oscillations, has also size-induced anomalies - e.g., for $\mathrm{Al}$ - that are not described by simple models. Furthermore, it was found that, at the melting, the diffusion of surface vacancies into the bulk is more beneficial for clusters

ISSN 2071-0194. Ukr. J. Phys. 2017. Vol. 62, No. 9 
with unfilled electron shells than for clusters with the magic number of atoms [9]. Those facts stimulate the enhanced interest to understanding the phase transition from the solid state into the liquid one as a configurational excitation of vacancies in the clusters.

From the thermodynamic viewpoint [12-14], the vacancy formation energy near the melting temperature should be lower for smaller clusters, whereas the vacancy concentration should be independent of the cluster size. However, thermodynamics gives no instructions concerning the mechanism of vacancy formation, so that the issues about the size dependence of the vacancy formation energy, vacancy concentration, and connection between vacancies and the melting process remain open.

Despite that the energy parameters of continuous (defect-free) metal clusters were calculated many times and in various models (see, e.g., works [1517] and the references therein), self-consistent calculations concerning the vacancy formation energy in clusters and how the quantization of the electron spectrum affects this quantity have not been performed till now. Moreover, one of the challenging problems is to study the size effect for the ionization potential of clusters containing a monovacancy. The obtained dependences can be useful for the analysis of the results of photoionization experiments and for the determination of the size dependence of the vacancy concentration, in particular, near the melting temperature.

The aim of this work was to calculate the energy characteristics of clusters with a vacancy in the framework of the stabilized jellium model using the KohnSham method and to analyze the size behavior of the vacancy formation energy, as well as its dependence on how this vacancy was formed.

\section{Basic Relations}

Let us consider a spherical metal cluster. We will compare the parameters of the spheres with the same number of atoms, $N$, at zero temperature. The radii of the solid spheres, $R_{N}$, and the spheres with a monovacancy at the center, $R_{N, v}$, are different:

$$
\begin{aligned}
& R_{N}=N^{1 / 3} r_{0}, \\
& R_{N, v}=(N+1)^{1 / 3} r_{0},
\end{aligned}
$$

where $r_{0}$ is the radius of a unit (i.e. Wigner-Seitz) cell per one atom.

ISSN 2071-0194. Ukr. J. Phys. 2017. Vol. 62, No. 9
In the framework of the stabilized jellium model, a monovacancy looks like a spherical neutral hole with the radius $r_{0}$ embedded into a homogeneous positively charged background. Using the Heaviside $\theta$-function, the distribution of the ionic charge in a cluster with a vacancy can be written in the form

$\rho_{v}(r)=\bar{n} \theta\left(r-r_{0}\right) \theta\left(R_{N, v}-r\right)$,

where $\bar{n}=3 Z /\left(4 \pi r_{0}^{3}\right)$ is the electron concentration in a uniform electron gas, and $Z$ the metal valency. For a continuous (defect-free) cluster, $\rho(r)=\bar{n} \theta\left(R_{N}-r\right)$.

The total energy of a metal sphere with a centered vacancy is written in the form of a functional of the electron concentration $n_{v}(r)$,

$E_{N, v}=T_{s, v}+\frac{e}{2} \int d^{3} r \phi_{v}(r)\left[n_{v}(r)-\rho_{v}(r)\right]+$
$+\int d^{3} r n_{v}(r) \varepsilon_{\mathrm{xc}}(r)-\Delta \bar{\varepsilon} \int d^{3} r \rho_{v}(r)+$
$+\langle\delta v\rangle_{\mathrm{WS}} \int d^{3} r \theta\left(r-r_{0}\right) \theta\left(R_{N, v}-r\right) n_{v}(r)$,

where $e>0$ is the elementary charge, and

$T_{s, v}=\sum_{i=1}^{N_{e}} \varepsilon_{i, v}-\int d^{3} r n_{v}(r) v_{\mathrm{eff}, v}(r)$

is the kinetic energy of $N_{e}=Z N$ noninteracting electrons.

The spatial distribution of the electrostatic potential, $\phi_{v}(r)$, is determined from the Poisson equation

$\nabla^{2} \phi_{v}(r)=-4 \pi e\left[n_{v}(r)-\rho_{v}(r)\right]$

under the condition

$\int_{0}^{\infty} d r 4 \pi r^{2}\left[\rho_{v}(r)-n_{v}(r)\right]=Q / e$,

where $Q$ is the total charge of the cluster.

In the stabilized jellium model [18], the energy per electron in bulk, $\bar{\varepsilon}$, consists of the unstabilized jellium component $\bar{\varepsilon}_{\mathrm{J}}$, Madelung energy $\varepsilon_{\mathrm{M}}$, and pseudopotential contribution $\bar{w}_{\mathrm{R}}$ :

$$
\begin{aligned}
& \bar{\varepsilon}=\bar{\varepsilon}_{\mathrm{J}}+\Delta \bar{\varepsilon}, \quad \bar{\varepsilon}_{\mathrm{J}}=\bar{t}_{s}+\bar{\varepsilon}_{\mathrm{xc}}, \\
& \Delta \bar{\varepsilon}=\varepsilon_{\mathrm{M}}+\bar{w}_{\mathrm{R}}, \quad\langle\delta v\rangle_{\mathrm{WS}}=\tilde{\varepsilon}+\Delta \bar{\varepsilon} \\
& \bar{w}_{\mathrm{R}}=2 \pi e^{2} \bar{n} r_{c}^{2}, \quad \varepsilon_{\mathrm{M}}=-\frac{9 Z e^{2}}{10 r_{0}}, \quad \tilde{\varepsilon}=-\frac{2}{3} \varepsilon_{\mathrm{M}},
\end{aligned}
$$



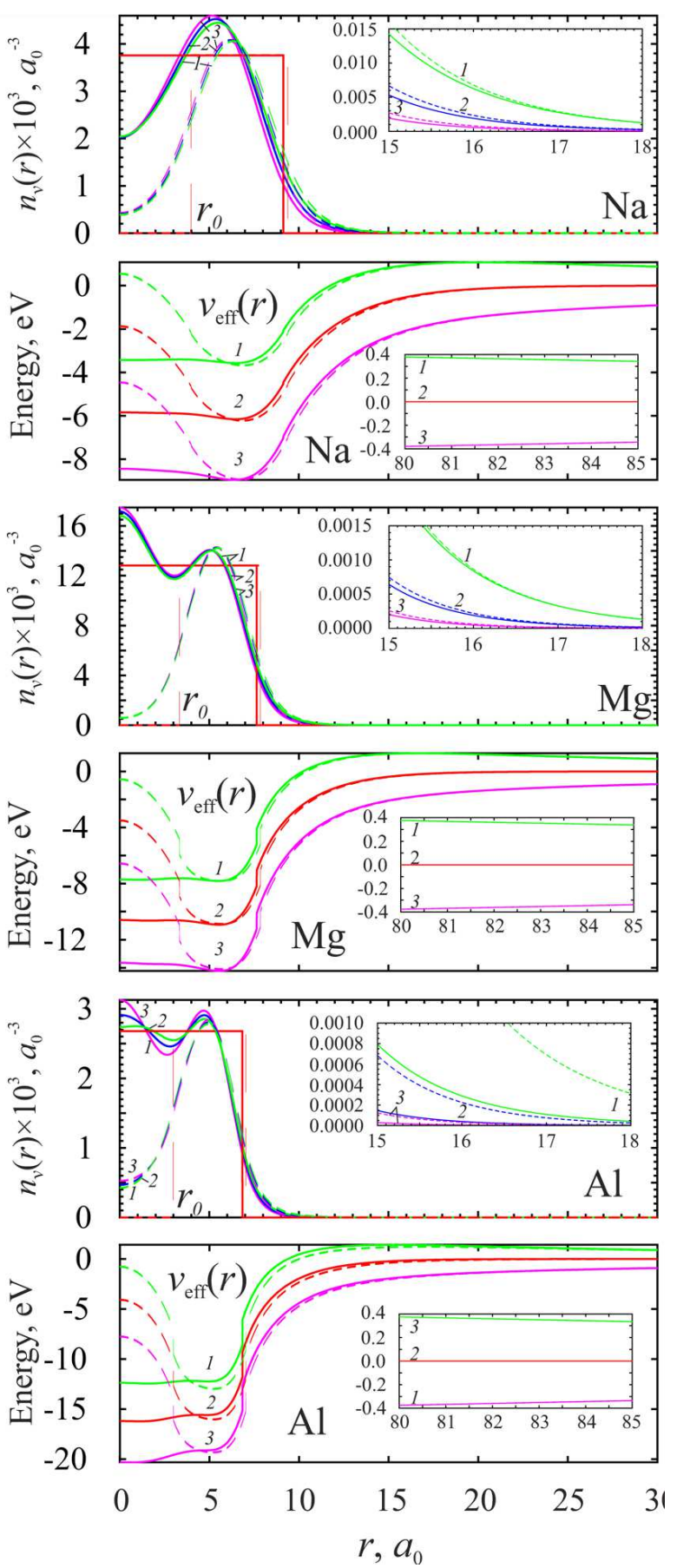

Fig. 1. Self-consistent profiles of the electron concentration and effective potential distributions in a defect-free cluster (solid curves) and a cluster with a centered vacancy (dashed curves). The both clusters contain the same number of atoms, $N=12$. The excess charge of the cluster $Q=-e(1), 0$ (2) and $+e(3)$ where $\bar{t}_{s}$ and $\bar{\varepsilon}_{\mathrm{xc}}$ are the kinetic and exchangecorrelation energies, respectively; $\bar{w}_{\mathrm{R}}$ is the difference between the ion pseudopotential and the electrostatic potential of the positively charged homogeneous background averaged over a Wigner-Seitz cell; $r_{c}$ the radius of Ashcroft pseudopotential; $\varepsilon_{\mathrm{M}}$ the Madelung energy of point-like ions with the valency $Z$ imbedded into the homogeneous negatively charged background; and $\tilde{\varepsilon}$ the intrinsic electrostatic energy of the homogeneous negatively charged background in the cell (see also work [19]).

In the Kohn-Sham method, the profile of the electron concentration distribution $n_{v}(r)$ in the cluster with a vacancy is expressed with the use of oneelectron wave functions,

$n_{v}(r)=\sum_{i=1}^{N}\left|\psi_{i, v}(r)\right|^{2}$.

The latter, together with the energy eigenvalues $\varepsilon_{i, v}$, are determined by solving the system of Kohn-Sham equations

$-\frac{\hbar^{2}}{2 m} \nabla^{2} \psi_{i, v}(r)+v_{\mathrm{eff}, v}(r) \psi_{i, v}(r)=\varepsilon_{i, v} \psi_{i, v}(r)$

with the effective one-electron potential

$v_{\mathrm{eff}, v}(r)=e \phi_{v}(r)+v_{\mathrm{xc}}(r)+$
$+\langle\delta v\rangle_{\mathrm{WS}} \theta\left(r-r_{0}\right) \theta\left(R_{N, v}-r\right)$.

This potential includes the electrostatic, $\phi_{v}(r)$, and exchange-correlation, $v_{\mathrm{xc}}\left[n_{v}(r)\right]$, potentials in the local density approximation (LDA). The energy is reckoned from the vacuum level, i.e. from the energy of an immovable electron located at a large distance from the specimen $\left(r \gg R_{N, v}\right)$, where the external charges are absent.

The system of Kohn-Sham equations together with the Poisson equation was solved numerically, by using the Numerov method. The increment step for the variable $r$ was approximately equal to 0.002 times the Bohr radius $a_{0}$.

In Fig. 1, the profiles of the electron concentration and effective potential distributions calculated for a continuous cluster and a cluster with a centered vacancy, each containing the same number of atoms $(N=12)$, are depicted. The radii of vacancies and clusters are indicated in the figure: $Z=1$ and 
$r_{0}=3.99 a_{0}$ for $\mathrm{Na}, Z=2$ and $r_{0}=3.34 a_{0}$ for $\mathrm{Mg}$, and $Z=3$ and $r_{0}=2.99 a_{0}$ for $\mathrm{Al}$. The radii of continuous and defect clusters with the same $N$ are different in accordance with determination (1). The insets in the figure demonstrate the profiles of electron concentrations and potentials at large distances from the clusters. Despite that the electron concentrations quickly decrease, the potential tails extend far away from the cluster (the calculation was carried out up to $\left.r \approx R_{N}+900 a_{0}\right)$. For the charged clusters, the electrostatic potential asymptotically falls down outside the cluster surface as $1 / r$. For large clusters, the spatial distribution of the electron concentration becomes similar to that near the surface of a semiinfinite metal and contains a considerable number of Friedel oscillations. The obtained profiles allow the total energy of the cluster to be calculated [see Eq. (3)].

\section{Ionization Potential and Electron Affinity}

Atomic clusters possess a structural periodicity, which is not a translational one, but looks like a "spherical periodicity" associated with the spherical layers of atoms (the coordination spheres). Analogously, for the clusters with a vacancy, it is possible to introduce the minimum number of atoms, $N=12$, corresponding to the spherical geometry of the problem.

Another peculiarity is only typical of metal clusters. It is associated with the filling degree of electron shells, as the number of atoms increases. In particular, clusters with filled electron shells are characterized by an enhanced stability in comparison to clusters with other sizes and partially filled shells. As $N \rightarrow \infty$, the difference between the magic and nonmagic clusters disappears.

The ionization potential (IP) and the electron affinity (EA) are defined as the differences of total energies

$$
\begin{aligned}
& \mathrm{IP}_{N, v}=E_{N, v}^{N_{e}-1}-E_{N, v}^{N_{e}}, \\
& \mathrm{EA}_{N, v}=E_{N, v}^{N_{e}}-E_{N, v}^{N_{e}+1},
\end{aligned}
$$

where $E_{N, v}^{N_{e}-1}$ and $E_{N, v}^{N_{e}+1}$ are the energies of a sphere with the radius $R_{N, v}$ and the excess charges $Q=e$ and $-e$, respectively ; and $E_{N, v}^{N_{e}}$ is the energy of a neutral sphere $(Q=0)$. In Fig. 2, the calculated dependences of IP and EA on $N^{-1 / 3}$ are shown. They allow the difference between the continuous and de- fect clusters to be traced, and a comparison with experimental data to be made. As the parameter $N$ increases above 12 , this difference can reach $0.1-0.5 \mathrm{eV}$ for $\mathrm{Na}$, and approximately twice as large for $\mathrm{Al}$. The letters s, p, d, f, g, h, i, j, k, and l correspond to the orbital numbers $l=0$ to 9 , respectively. The maximum difference between the continuous and defect clusters is observed, when the transition from a completely filled shell to an empty one takes place. With the increase of $N$, this difference disappears.

For the clusters with a monovacancy, the vacancy concentration $c_{v}=1 / N$. From whence, we obtain the relation $N^{-1 / 3}=c_{v}^{1 / 3}$, with $c_{v} \rightarrow 0$ as $N \rightarrow \infty$. In the case where the vacancy is not single but multiple, but the vacancy concentration is low (so that the vacancies do not interact with one another), our figures can be used to qualitatively trace the dependences of energy characteristics on the vacancy concentration.

Figure 2 also exhibits the asymptotics of IP and EA,

$$
\begin{aligned}
& \mathrm{IP}_{N}=-\mu_{0}+\alpha e^{2} / R_{N}, \\
& \mathrm{EA}_{N}=-\mu_{0}-\beta e^{2} / R_{N},
\end{aligned}
$$

which are written down in accordance with the expansion of the electron chemical potential in a series in $R_{N}^{-1}$ :

$$
\mu\left(R_{N}\right)=\mu_{0}+\mu_{1} / R_{N}+O\left(R_{N}^{-2}\right),
$$

where $\mu_{0}=-W_{0}$, and $W_{0}$ is the work function of a metal as $R_{N} \rightarrow \infty$. The coefficients $\alpha=1 / 2-\mu_{1} / e^{2}$ and $\beta=1 / 2+\mu_{1} / e^{2}$ contain the parameter $\mu_{1}=$ $=2 \sigma_{0} / \bar{n}$, which is typical of every material; and $\sigma_{0}$ is the specific energy of a plane surface $(N \rightarrow \infty)$ [20,21].

The ionization potential and the electron affinity demonstrate a strongly oscillating behavior, which is associated with the spherical shell structure. They asymptotically tend to $W_{0}$ rather slowly, which is a result of the orbital degeneration and large angular quantum numbers $l$. Experimental oscillations are considerably weaker. If the calculations are carried out in the local spin density approximation (LSDA), the amplitude of oscillations is smaller.

Using Koopmans' theorem, formulas (10) can be rewritten in the form

$$
\begin{aligned}
& \mathrm{IP}_{N, v}=-\varepsilon_{N, v}^{\mathrm{HO}}+\frac{e^{2}}{2 C_{N, v}^{+}}, \\
& \mathrm{EA}_{N, v}=-\varepsilon_{N, v}^{\mathrm{LU}}-\frac{e^{2}}{2 C_{N, v}^{-}},
\end{aligned}
$$



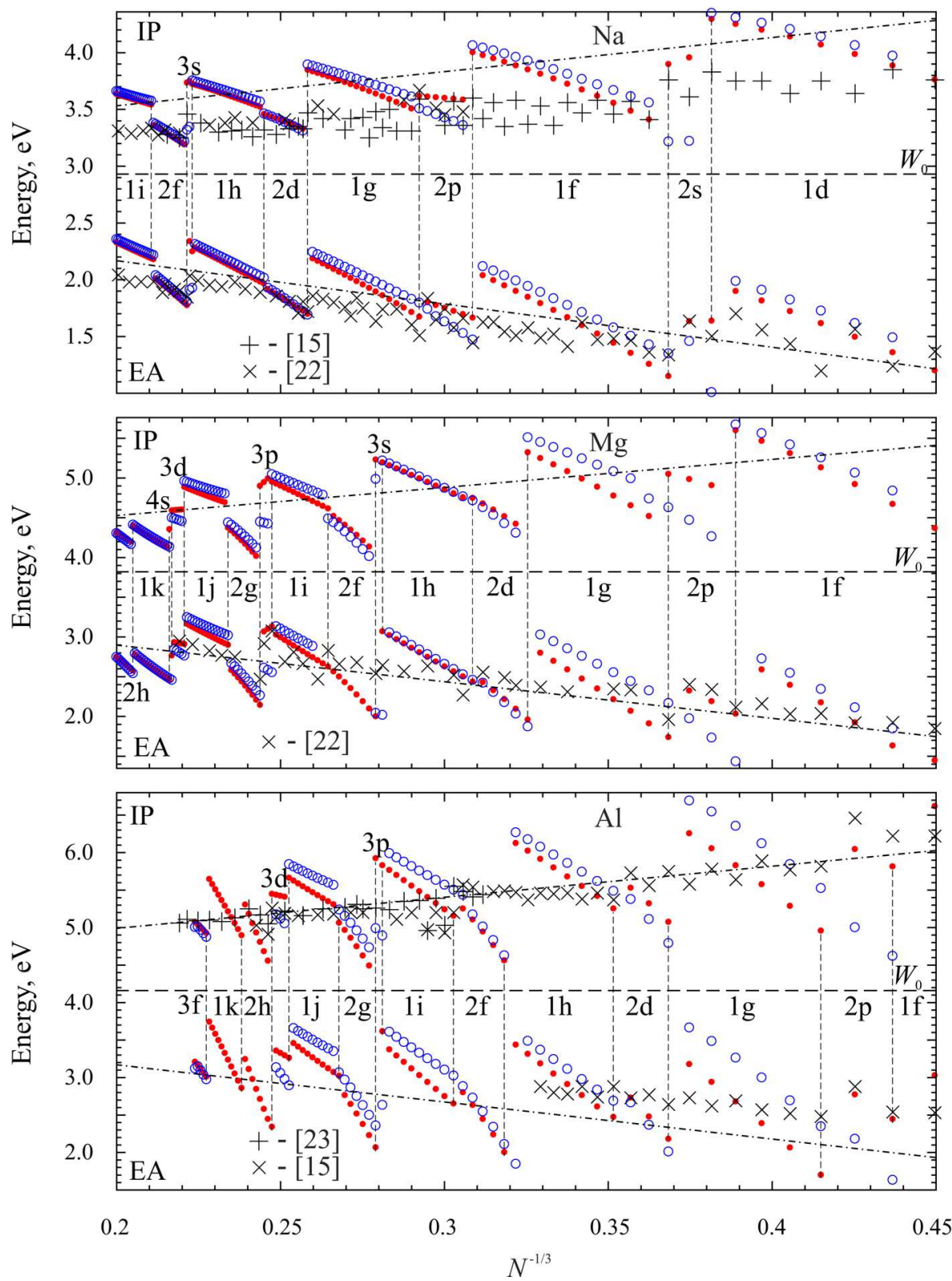

Fig. 2. Ionization potential and electron affinity calculated by formulas (10) for continuous clusters $(\bullet)$ and clusters with a monovacancy $(\circ)$. Symbols + and $\times$ correspond to experimental data. Asymptotics (12) are shown by dash-dotted curves

where $\varepsilon_{N, v}^{\mathrm{HO}}$ and $\varepsilon_{N, v}^{\mathrm{LU}}$ are the energies of the highest occupied and lowest unoccupied, respectively, electron orbitals of the cluster, and $C_{N, v}^{ \pm}$the corresponding electric capacities.

Figure 3 illustrates effects associated with the filling of electron shells in continuous $\mathrm{Na}, \mathrm{Mg}$, and $\mathrm{Al}$ clusters, as the number of atoms in them grows. Dashes in the figure mark the filled electron levels, and points correspond to the empty (virtual) ones.

For partially filled shells, $\varepsilon_{N, v}^{\mathrm{HO}}=\varepsilon_{N, v}^{\mathrm{LU}} \approx \mu\left(R_{N, v}\right)$. The maximum values of $\varepsilon_{N, v}^{\mathrm{HO}}$ correspond to completely occupied shells, whereas the magic atomic numbers $N^{*}$ for continuous spherical clusters and 
spherical clusters with a vacancy do not coincide in all cases. For $\mathrm{Na}$, the obtained values for $N^{*}$ are 2, 8, 18, 20, 34, 40, 58, 68, 90, 92, 106, 132, 138, 168, 186, 196, 198, (230), 232, (252), and 254. For $\mathrm{Mg}, N^{*}=4$, $9,10,17,20,29,34,45,46,53,66,69,78,93,98,(99)$, 115, (116), 126, 127, 134, 153, 156, 169, 178, 199, 204, and 219. For Al, $N^{*}=6,(30), 44,46,52,62,\{66\}$ (84), (102), \{104\}, \{136\}, 146, \{154\}, (180), (202), and 204. The numbers in parentheses mean those $N^{*}$ values for defect clusters that do not coincide with the corresponding values for continuous clusters, and the numbers in braces mean the inverse case.

With the growth of $R_{N, v}$, the quantities $-\varepsilon_{N, v}^{\mathrm{HO}}$ and $-\varepsilon_{N, v}^{\mathrm{LU}}$ oscillate, by approaching $-\mu\left(R_{N, v}\right)$ as $R \rightarrow \infty$. Their oscillation amplitudes decrease as approximately $1 / R_{N, v}^{3}$.

Let us come back to Fig. 2 and introduce the notation $\Delta\left(\mathrm{IP}_{N}\right)=\mathrm{IP}_{N, v}-\mathrm{IP}_{N}$. At first glance, the positive sign of $\Delta\left(\mathrm{IP}_{N}\right)$ (the circles in the figure are located above the points for the same $N$ ) seems unexpected. Exceptions include clusters with such $N$ values, at which the maximum contribution is provided by levels with small $l(s-, p$-, and, partially, $d$ orbitals). In Fig. 2, those narrow intervals are located between vertical dash-dotted lines.

The main vacancy dependence of the asymptotic behavior of $\mathrm{IP}_{N, v}$ and $\mathrm{IP}_{N}$ is contained in the work function $W_{0}\left(c_{v}\right)<W_{0}\left(c_{v}=0\right)$ [24]. Therefore, it was supposed that $\Delta\left(\mathrm{IP}_{N}\right)<0$ every time.

In the case of small clusters with a monovacancy, the perturbation from vacancies with the concentration $c_{v} \sim 1 / R_{N}^{3}$ becomes considerable. As follows from the behavior of $v_{\text {eff, } v}(r)$ in Fig. 1, electrons are "squeezed out" by the vacancy from the cluster center toward the surface, and they are mainly accumulated in a spherical layer with $r_{0}<r<R_{N}$. When integrating in Eq. (3) in the spherical coordinates, this is a region that gives the main contribution to the energy. This fact is confirmed by the spectral values of energies that correspond to points (circles) in Fig. 2. As an example, the values of $\varepsilon_{n_{r}, l}$, where $n_{r}$ and $l$ are the radial and orbital quantum numbers, respectively, are quoted below. For $\mathrm{Na}_{12}$ : $\varepsilon_{n_{r}=0, l=0}=-4.925,(-4.577) \mathrm{eV} ; \varepsilon_{0,1}=-3.871$, $(-3.831) \mathrm{eV}$; and $\varepsilon_{0,2}^{\mathrm{HO}, \mathrm{LU}}=-2.595,(-2.708) \mathrm{eV}$. For $\mathrm{Na}_{18}: \varepsilon_{0,0}=-5.073,(-4.755) \mathrm{eV} ; \varepsilon_{0,1}=-4.177$, $(-4.135) \mathrm{eV} ; \varepsilon_{0,2}^{\mathrm{HO}}=-3.119,(-3.189) \mathrm{eV} ;$ and $\varepsilon_{1,0}^{\mathrm{LU}}=$ $=-2.787,(-2.048) \mathrm{eV}$.

ISSN 2071-0194. Ukr. J. Phys. 2017. Vol. 62, No. 9
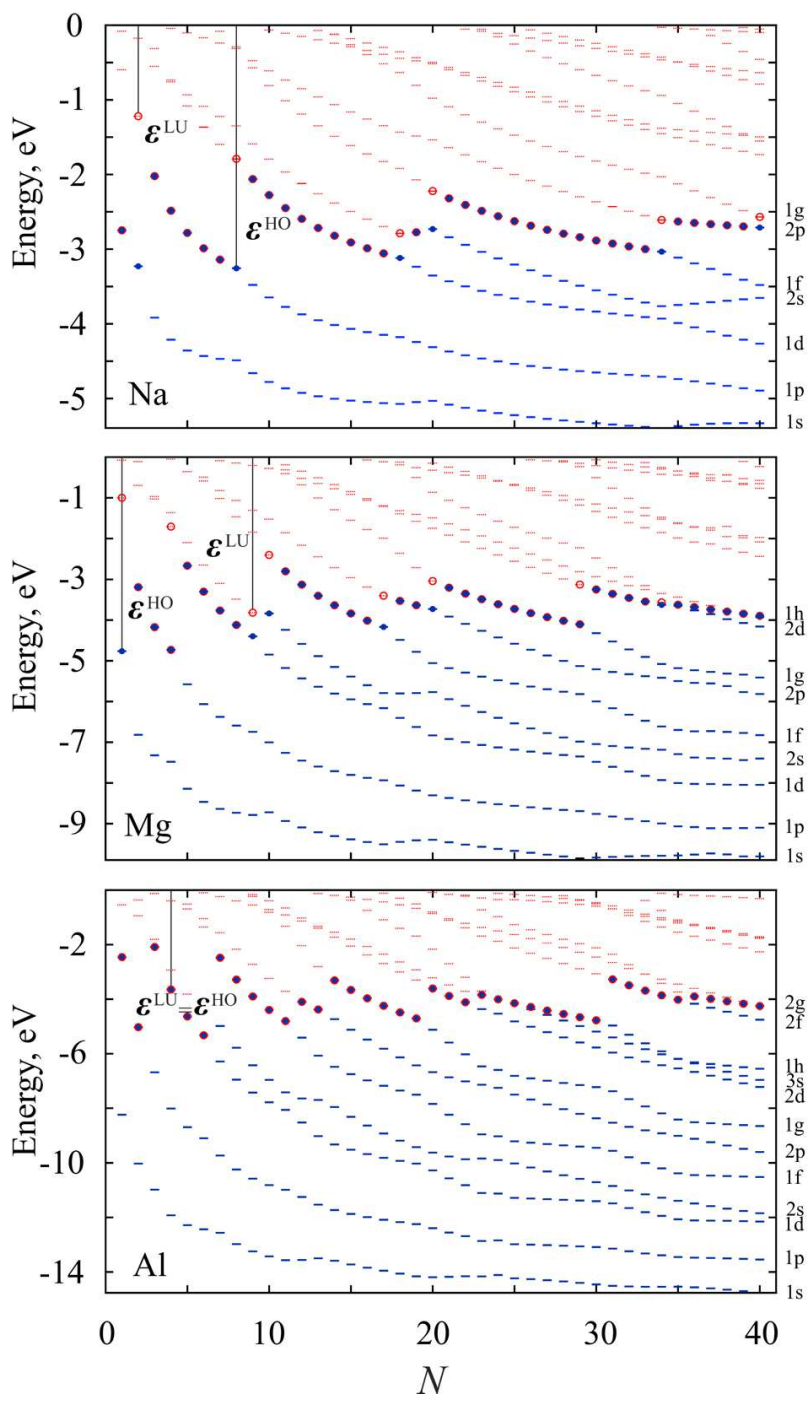

Fig. 3. Spectra of continuous clusters. The highest occupied, $\varepsilon_{N}^{\mathrm{HO}}(\bullet)$, and the lowest unoccupied, $\varepsilon_{N}^{\mathrm{LU}}(\mathrm{\circ})$, energy levels are marked

With the growth of $N$, the contribution of the cluster bulk becomes more substantial, so that points and circles in the asymptotics in Fig. 2 switch their positions. In other words, the difference $\Delta\left(\mathrm{IP}_{N \rightarrow \infty}\right)$ becomes negative.

The self-consistent values of IP, EA, $\varepsilon^{\mathrm{HO}}$, and $\varepsilon^{\mathrm{LU}}$ calculated by formulas (10) make it possible to use expressions (10) in order to calculate the capacitances

$C_{N, v}^{+}=\frac{e^{2}}{2\left(\operatorname{IP}_{N, v}+\varepsilon_{N, v}^{\mathrm{HO}}\right)}$, 

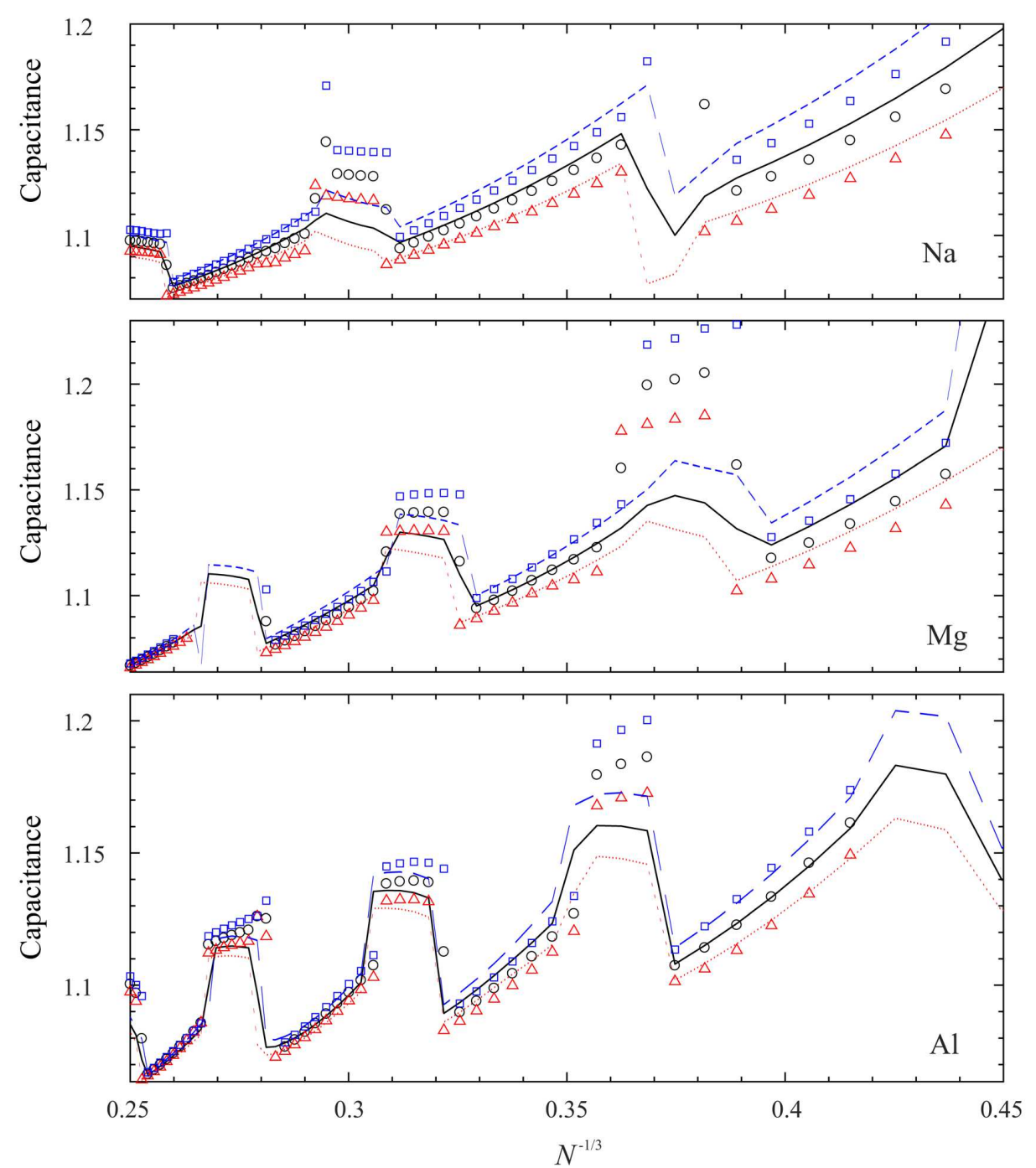

Fig. 4. Normalized capacitances of continuous clusters (the dotted, solid, and dashed curves correspond to positively charged, neutral, and negatively charged clusters, respectively) and clusters with a monovacancy (symbols $\Delta, \circ$, and $\square$ correspond to positively charged, neutral, and negatively charged clusters, respectively) calculated by formulas (13)

$C_{N, v}^{-}=\frac{-e^{2}}{2\left(\mathrm{EA}_{N, v}+\varepsilon_{N, v}^{\mathrm{LU}}\right)}$,

$C_{N, v}^{\mathrm{eff}}=\frac{e^{2}}{\mathrm{IP}_{N, v}+\varepsilon_{N, v}^{\mathrm{HO}}-\mathrm{EA}_{N, v}-\varepsilon_{N, v}^{\mathrm{LU}}}$.

Analogous formulas for $C_{N}$ correspond to defectfree clusters.

In classical electrostatics, the capacitances of conducting spheres are determined by their radii $R_{N, v}$. The surface roughness on the atomic scale (atoms have a finite volume) does not allow the boundary to be established accurately [25]. In the jellium model, the boundary of the ionic frame is always located at the coordinate $r=R_{N, v}$. However, if the frame radius $R_{N, v}$ decreases, the electron cloud goes more and more beyond the frame limits. Moreover, such a "spilling out"depends on the sign of the cluster excess charge (Fig. 1). In this connection, the quantities $C_{N, v}^{\text {eff }}, C_{N, v}^{+}$, and $C_{N, v}^{-}$are equal to one another only in the $N \rightarrow \infty$ limit.

Figure 4 demonstrates the results of calculations obtained for the capacitances $C_{N}$ and $C_{N, v}$ of the 
$\mathrm{Na}, \mathrm{Mg}$, and $\mathrm{Al}$ clusters normalized to the radius $R_{N}$ or $R_{N, v}$, respectively (in atomic units). The largest difference is observed in the intervals of $N$, where the $s$ - and $p$-electron shells become filled. The signalternating difference

$C_{N, v} / R_{N, v}-C_{N} / R_{N}$

is mainly determined by the ratio between the quantities $\varepsilon^{\mathrm{HO}}$ and $\varepsilon^{\mathrm{LU}}$ for various $l$ in the continuous and defect clusters, and this ratio can be changed depending on the principal quantum number. The capacitance of defect clusters, when their shells with small $l$ are filled, is larger than that of continuous ones. For large $l$, the situation is inverse.

For a $\mathrm{Na}$ atom $(N=1)$, using the corresponding experimental data $\left(\mathrm{IP}_{1}=5.14 \mathrm{eV}, \mathrm{EA}_{1}=0.55 \mathrm{eV}\right.$, and $R_{1}=r_{0}$ ), as well as the condition $\varepsilon_{1}^{\mathrm{HO}}=\varepsilon_{1}^{\mathrm{LU}}$ for the unfilled shells, we obtain the test value $C_{1}^{\text {eff }} / r_{0}=$ $=1.8$. This value agrees well with the values calculated for the smallest clusters. In the case of unclosed electron shells, the cluster can posess a lower symmetry, e.g., the spheroidal one.

The charging effect consists in that the electric capacitance of cluster anions and cations depends on the sign of excess charge. The normalization of the capacitances makes it possible to give a simple interpretation to the calculation results, Namely, the negative excess charge gives rise to an effective growth of the electron cloud (its radius) in the cluster, whereas the smaller number of electrons (the positive excess charge) to a reduction of both the radius and the capacitance. This conclusion is qualitatively confirmed by the behavior of electron profiles shown in Fig. 1 .

In work [26], besides the measurement of heat capacities for anions and cations in $\mathrm{Al}_{35-70}$ clusters, the ionization potential and the electron affinity were calculated. The calculations were performed, by using the density functional method and by accounting for the global minimum condition for the total energy in various atomic configurations. The corresponding results (see Fig. 9 in work [26]) were approximated by us in the form

$$
\begin{aligned}
& \mathrm{IP}_{N}=\left(4.17+3.97 N^{-1 / 3}\right) \mathrm{eV}, \\
& \mathrm{EA}_{N}=\left(3.88-3.05 N^{-1 / 3}\right) \mathrm{eV}
\end{aligned}
$$

Unfortunately, although the calculations were complicated, the presented expressions did not reproduce the work function and the value of $\mu_{1}$ in the coefficients $\alpha$ and $\beta$ in formula (11). We obtained $\mu_{1} \approx+0.067$ a.u. from $\operatorname{IP}_{N}$ and $\mu_{1} \approx-0.165$ a.u from $\mathrm{EA}_{N}$. It will be recalled that $\mu_{1} \approx+0.1$ a. $\mathrm{u}$. for $\mathrm{Na}$ [27, 28].

\section{Dissociation, Cohesive, and Vacancy Formation Energies}

The energy of dissociation of a neutral metal (Me) cluster in accordance with the reaction $\mathrm{Me}_{N} \rightarrow$ $\mathrm{Me}_{N-1}+\mathrm{Me}_{\text {at }}$ is determined as the difference of total energies

$\varepsilon_{N}^{\mathrm{dis}}=\left[E_{N-1}+E_{\mathrm{at}}\right]-E_{N}=N \varepsilon_{N}^{\mathrm{coh}}-(N-1) \varepsilon_{N-1}^{\mathrm{coh}}$.

In the stabilized jellium model, the energy of the atom, $E_{\text {at }}$, is the total energy of a metal sphere with the radius $r_{0}$.

The dissociation energy of metal ions in the clusters and the ionization potential of clusters were measured repeatedly and commented in detail $[15,22,23,29$, $30]$. Those data are traditionally used to calculate the cohesive energy of neutral clusters.

By definition, the cohesive energy $\varepsilon_{N}^{\text {coh }}$ is the binding energy of atoms in a cluster per one atom. The cohesive energy $\varepsilon_{N}^{\text {coh }}$ is determined as the difference between the cumulative energy of $N$ free atoms and the energy of the cluster consisting of $N$ atoms:

$\varepsilon_{N}^{\mathrm{coh}}=\left(N E_{\mathrm{at}}-E_{N}\right) / N=E_{\mathrm{at}}-E_{N} / N$.

If $N \rightarrow \infty$, then $\varepsilon_{N}^{\text {coh }} \rightarrow \varepsilon_{\infty}^{\text {coh }} \equiv \varepsilon^{\text {coh }}\left(r_{0}\right)$. The values $\varepsilon^{\mathrm{coh}}\left(r_{0}\right)=3.97$ and $1.16 \mathrm{eV}$ calculated by us for $\mathrm{Al}$ and $\mathrm{Na}$, respectively, agree well with the experimental values $\varepsilon_{\infty}^{\text {coh }}=3.39$ and $1.11 \mathrm{eV}$, respectively (see work [31] and the references therein).

The coupling equation looks like

$\varepsilon_{N}^{\mathrm{coh}}=\frac{1}{N} \sum_{n=2}^{N} \varepsilon_{n}^{\mathrm{dis}}$.

The asymptotics of the cluster-size dependence of the cohesive energy (15) is a well-known result [1]

$\varepsilon_{N}^{\mathrm{coh}}=\varepsilon^{\mathrm{coh}}\left(r_{0}\right)-\frac{2 \sigma_{0}}{n_{\mathrm{at}} R_{N}}$,

where the last term can be rewritten in the form $-Z \mu_{1} / R_{N}$.

Note that it was marked as long ago as in works by Frenkel and Langmuir that the following universal 
relation is valid for some substances at low temperatures:

$4 \pi r_{0}^{2} \sigma / q \approx 2 / 3$

This relation includes the following observable quantities: the average distance $r_{0}$ between the atoms, the specific surface energy $\sigma$, and the evaporation heat $q$ (see the table in work [25]). Using this relation, asymptotics (17) can be rewrittren in the form that is convenient for making estimations:

$\varepsilon_{N}^{\mathrm{coh}} \approx \varepsilon^{\mathrm{coh}}\left(r_{0}\right)\left[1-(4 / 9) N^{-1 / 3}\right]$.

Then, using formulas (17) and (14), it is easy to reveal the convergence of the asymptotics for $\varepsilon_{N}^{c o h}$ and $\varepsilon_{N}^{\mathrm{dis}}$.

It is of interest to determine the influence of the charging on the cohesive energy of clusters. Using definition (15), the notations $E_{N}^{+} \equiv E_{N}^{N_{e}-1}, E_{N}^{-} \equiv$ $\equiv E_{N}^{N_{e}+1}$, and $\varepsilon_{N}^{\mathrm{coh}, \pm}=E_{\mathrm{at}}-E_{N}^{ \pm} / N$, and formula (10), the difference between the cohesive energies for the charged and neutral clusters can be determined as

$$
\begin{aligned}
& \Delta \varepsilon_{N}^{\mathrm{coh},+} \equiv \varepsilon_{N}^{\mathrm{coh},+}-\varepsilon_{N}^{\mathrm{coh}}=-\frac{1}{N} \mathrm{IP}_{N}, \\
& \Delta \varepsilon_{N}^{\mathrm{coh},-}=\frac{1}{N} \mathrm{EA}_{N} .
\end{aligned}
$$

In Figs. 5 and 6, the dependences of the dissociation and cohesive, respectively, energies on the parameter $N$ are compared between the continuous and defect (neutral and charged) clusters. It should be noted that the positive or negative excess charge results in a reduction or growth, respectively, of the dissociation and cohesive energies.

The size dependence of the dissociation energy exhibited in Fig. 5 includes quantum oscillations around the asymptotics. The values of $\varepsilon_{N, v}^{\text {dis }}$ for a defect cluster are larger at large $l$ and smaller at small $l$ than those for a continuous cluster. For the continuous and defect clusters with the same number of atoms $N$, besides a change in the filling order of electron levels, a substantial difference between the behavior of the dissociation energy should be emphasized: for small $l$, the dissociation energy of continuous clusters decreases and that of defect ones increases with the growth of $N$. A comparison of the data depicted in Figs. 5 and 6 confirms the accuracy of formula (16) and explains a distinction in the arrangement of local maxima in those figures. The calculated dependences of $\varepsilon_{N, v}^{\text {coh }}$ are closer to the experimental values obtained at $T=150 \mathrm{~K}[30]$ than the dependences of $\varepsilon_{N}^{\text {coh }}$. Furthermore, the values of $\varepsilon_{N}^{\text {dis }}$ determined from the measurements of the melting temperature and the latent heat of the transition can be negative near the phase transition point [10].

Hence, a conclusion can be drawn that the most stable defect-free clusters are those, in which the levels with small $l$ are the last filled ones. In the case of defect clusters, the situation is inverse. In experiments, the size-induced oscillations of $\varepsilon_{N}^{\text {dis }}$ are most probaly suppressed by temperature effects (see Fig. 9 in work [29]).

As is shown in Fig. 6, the cohesive energy of anions and cations in charged clusters differs from that in neutral clusters. The presence of a positive excess charge results in a reduction of the cohesive energy owing to an increase of electrostatic repulsion forces, whereas the negative excess charge gives rise to the opposite effect. The behavior of the cohesive energy of ionized clusters is completely described by formula (18).

It is reasonable to suppose that the cluster melting temperature "traces" changes in the cohesive energy (more exactly, the dissociation energy), i.e. the cluster melting temperature can be controlled by means of the charging. A comparison of Fig. 5 with the experimental size dependences of the melting heats for anions and cations in $\mathrm{Al}_{35-70}$ clusters (see Fig. 4 in work [26]) testified to their correlation.

A considerable number of works were devoted to $a b$ initio calculations of the vacancy formation energy in metals [32]. In the stabilized jellium and liquid drop models, the cohesive energy of an atom and the vacancy formation energy are presented in the form of Padé expansions [31] (see also work [25]). In the notations of work [25], the results of work [31] can be presented as follows:

$$
\begin{aligned}
& \varepsilon^{\operatorname{coh}}\left(r_{0}\right)=4 \pi r_{0}^{2} \sigma_{0}\left(1+\delta_{1} / r_{0}+\delta_{2} / r_{0}^{2}\right), \\
& \varepsilon^{\operatorname{vac}}\left(r_{0}\right)=4 \pi r_{0}^{2} \sigma_{0}\left(1-\delta_{1} / r_{0}+\delta_{2} / r_{0}^{2}\right) .
\end{aligned}
$$

The values $\varepsilon_{\infty}^{\mathrm{vac}} \equiv \varepsilon^{\mathrm{vac}}\left(r_{0}\right)=0.33,0.73$, and $1.00 \mathrm{eV}$ calculated in work [33] for $\mathrm{Na}, \mathrm{Mg}$, and $\mathrm{Al}$, respectively, using the phases of electron wave scattering at a vacancy potential value agree with the corresponding experimental values of $0.335,084$, and $0.73 \mathrm{eV}[31]$.

Using the quantities $\varepsilon^{\operatorname{coh}}\left(r_{0}\right)$ and $\varepsilon^{\mathrm{vac}}\left(r_{0}\right)$, as well as the values $\delta_{2} / r_{0}^{2}=-0.13(\mathrm{Na}),-0.015(\mathrm{Mg})$, 

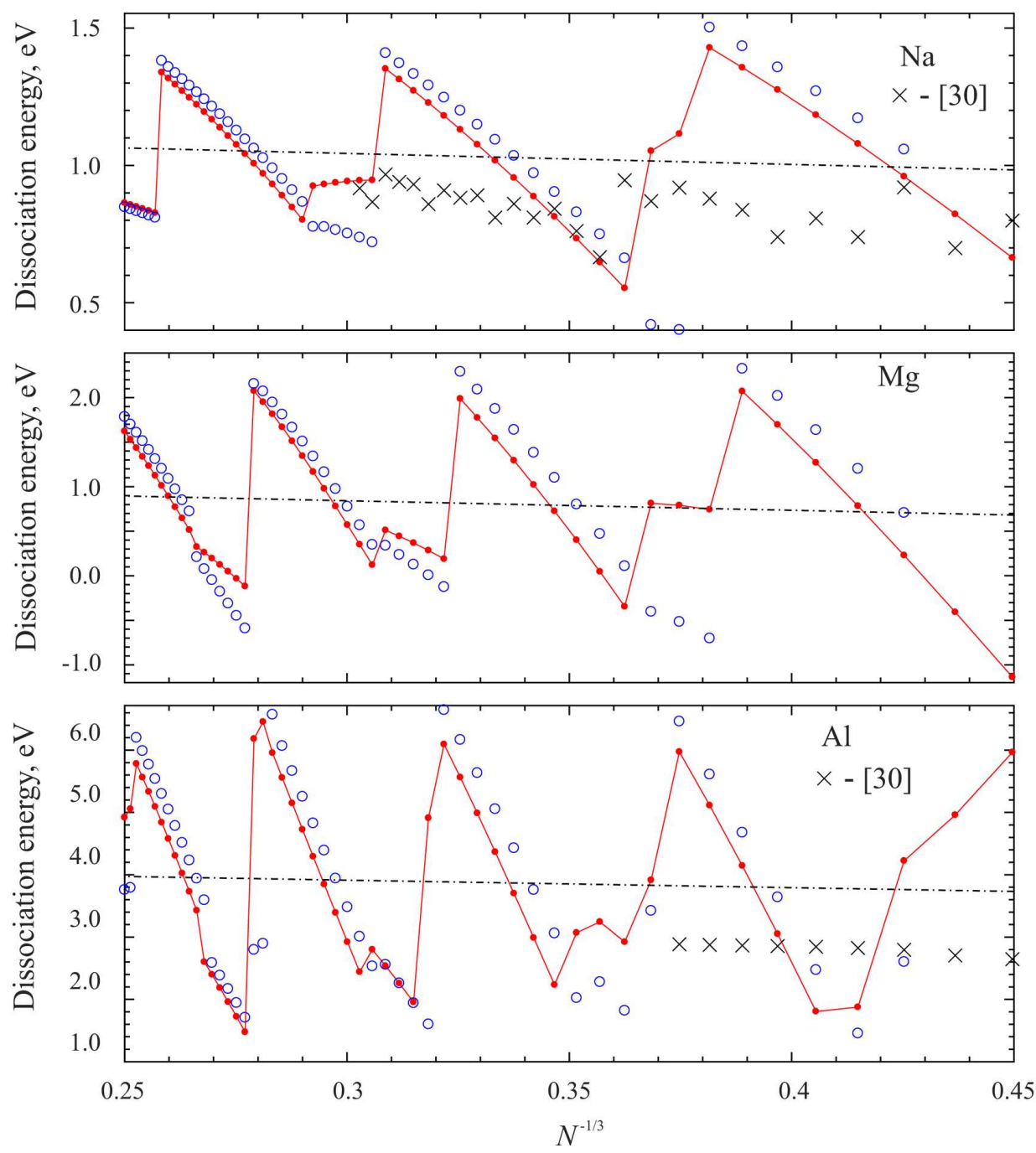

Fig. 5. Dissociation energies $\varepsilon^{\text {dis }}$ for neutral continuous $(\bullet)$ and defect $(\circ)$ clusters, and their asymptotics (17). Experimental values are shown by symbols $\times$

and $+0.22(\mathrm{Al})$ taken from work [31], we obtained $\delta_{1} / r_{0}=0.32$ for $\mathrm{Na}, 0.54$ for $\mathrm{Mg}$, and 0.57 for Al. The quantities $\delta_{1}$ and $\delta_{2}$ are required to construct the asymptotics for the vacancy formation energy.

No self-consistent calculations of $\varepsilon_{N, v}^{\mathrm{vac}}$ have been performed for clusters because of the necessity to work out the process of vacancy formation in detail. Therefore, it is important to compare the expedience of the vacancy formation driven by two mechanisms. According to the Schottky mechanism, an atom is evaporated from a continuous sphere. In the final state, there is a vacancy with the radius $r_{0}$ at ISSN 2071-0194. Ukr. J. Phys. 2017. Vol. 62, No. 9 the sphere center. In this case,

$\varepsilon_{N, v}^{\mathrm{vac}, \mathrm{Sh}}=\left[E_{N-1, v}+E_{\mathrm{at}}\right]-E_{N}=$

$=N \varepsilon_{N}^{\mathrm{coh}}-(N-1) \varepsilon_{N-1, v}^{\mathrm{coh}}$,

where $E_{N-1, v}$ is the energy of a sphere with a centered vacancy (the layer located between $r=r_{0}$ and $r=R_{N-1, v}$ contains $N-1$ atoms). The essence of the second mechanism [25], at which the number of atoms in the sphere does not change, consists in the "blowing" of a bubble-vacancy with the radius $r_{0}$ at the sphere center. In this case,

$\varepsilon_{N, v}^{\mathrm{vac}, \mathrm{blow}}=E_{N, v}-E_{N}=N\left(\varepsilon_{N}^{\mathrm{coh}}-\varepsilon_{N, v}^{\mathrm{coh}}\right)$. 

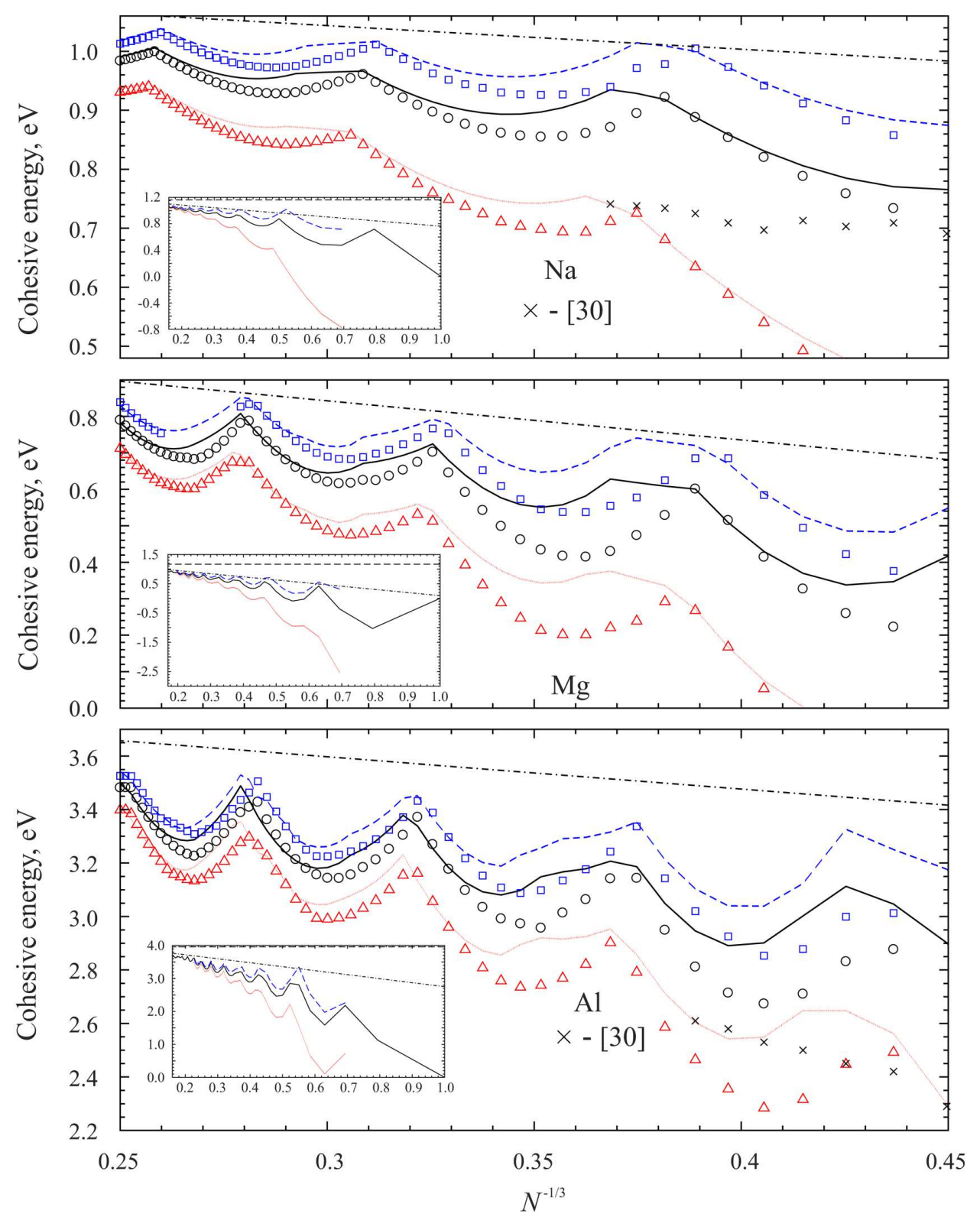

Fig. 6. Cohesive energy $\varepsilon^{\text {coh }}$ of continuous clusters (the dotted, solid, and dashed curves correspond to positively charged, neutral, and negatively charged clusters, respectively) and clusters with a monovacancy (symbols $\triangle, \circ$, and $\square$ correspond to positively charged, neutral, and negatively charged clusters, respectively). Asymptotics (17) is shown by a dash-dotted curve. Experimental values are shown by symbols $\times$. The corresponding results obtained for defect-free clusters in the whole interval of calculated $N$ are shown in the insets

A comparison between formulas (21) and (22) demonstrates the expedience of the second mechanism owing to the relation

$\varepsilon_{N, v}^{\mathrm{vac}, \mathrm{Sh}}=\varepsilon_{N, v}^{\mathrm{vac}, \mathrm{blow}}+\varepsilon_{N, v}^{\mathrm{dis}}$.
Let us analyze the asymptotics of the vacancy formation energy. Its size dependence is determined by the difference between the total energies of spheres calculated by formulas (21) and (22) as $N \rightarrow \infty$

ISSN 2071-0194. Ukr. J. Phys. 2017. Vol. 62, No. 9 

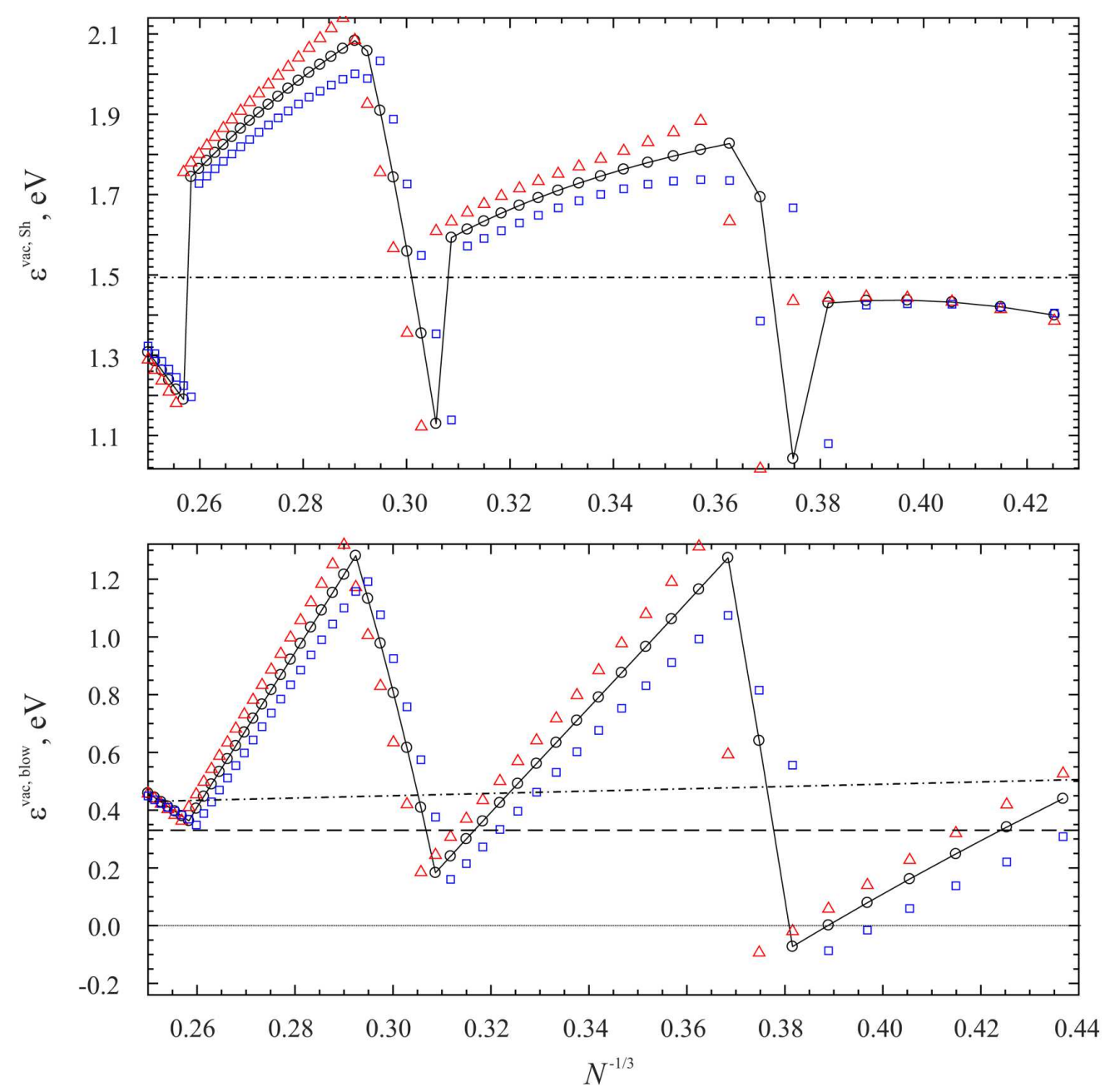

Fig. 7. Energies of the monovacancy formation $\varepsilon_{N, v}^{\mathrm{vac}, \mathrm{Sh}}(21)$ and $\varepsilon_{N, v}^{\mathrm{vac}, \text { blow }}(22)$ in Na. Symbols $\triangle, \circ$, and $\square$ correspond to positively charged, neutral, and negatively charged clusters, respectively. Asymptotics (24) and (23) are shown by dash-dotted curves. The horizontal dotted line corresponds to $\varepsilon_{\infty}^{\mathrm{vac}} \equiv \varepsilon^{\mathrm{vac}}\left(r_{0}\right)=0.33 \mathrm{eV}$

and is reduced to the difference between the total surface energies. In the case of the vacancy "blowing" mechanism, using relation (10) and the formula $R_{N, v}=R_{N}\left(1+\frac{1}{3} \zeta^{3}\right)$, where $\zeta \equiv r_{0} / R_{N} \ll 1$, and holding the required expansion order, we obtain

$\varepsilon_{N, v}^{\mathrm{vac}, \mathrm{blow}}=4 \pi R_{N, v}^{2} \sigma_{0}\left(1+\delta_{1} / R_{N, v}+\delta_{2} / R_{N, v}^{2}\right)+$

$+\varepsilon^{\mathrm{vac}}\left(r_{0}\right)-4 \pi R_{N}^{2} \sigma_{0}\left(1+\delta_{1} / R_{N}+\delta_{2} / R_{N}^{2}\right)=$

$=\varepsilon^{\mathrm{vac}}\left(r_{0}\right)\left(1+\frac{2}{3 N^{1 / 3}\left(1-\delta_{1} / r_{0}+\delta_{2} / r_{0}^{2}\right)}\right)$.

For the Schottky mechanism, in accordance with expression (23) and the formula $R_{N-1, v}=R_{N}$, the ISSN 2071-0194. Ukr. J. Phys. 2017. Vol. 62, No. 9 asymptotics is determined by the sum of expressions (24) and (17). The asymptotic dependence of $\varepsilon_{N, v}^{\text {vac,Sh }}$ weakly depends on $N$, whereas dependence (24) demonstrates a reduction of the vacancy formation energy with the growth of $N$. This behavior agrees with the conclusions of work [34], but contradicts the results of works [12-14].

Figures 7 to 9 exhibit the calculation results obtained for the energy of the vacancy formation driven by two mechanisms. The exhibited results confirm the validity of formula (23), namely, the expedience of the vacancy blowing. All dependences strongly oscillate. For some $N$ grouped into narrow intervals, espe- 

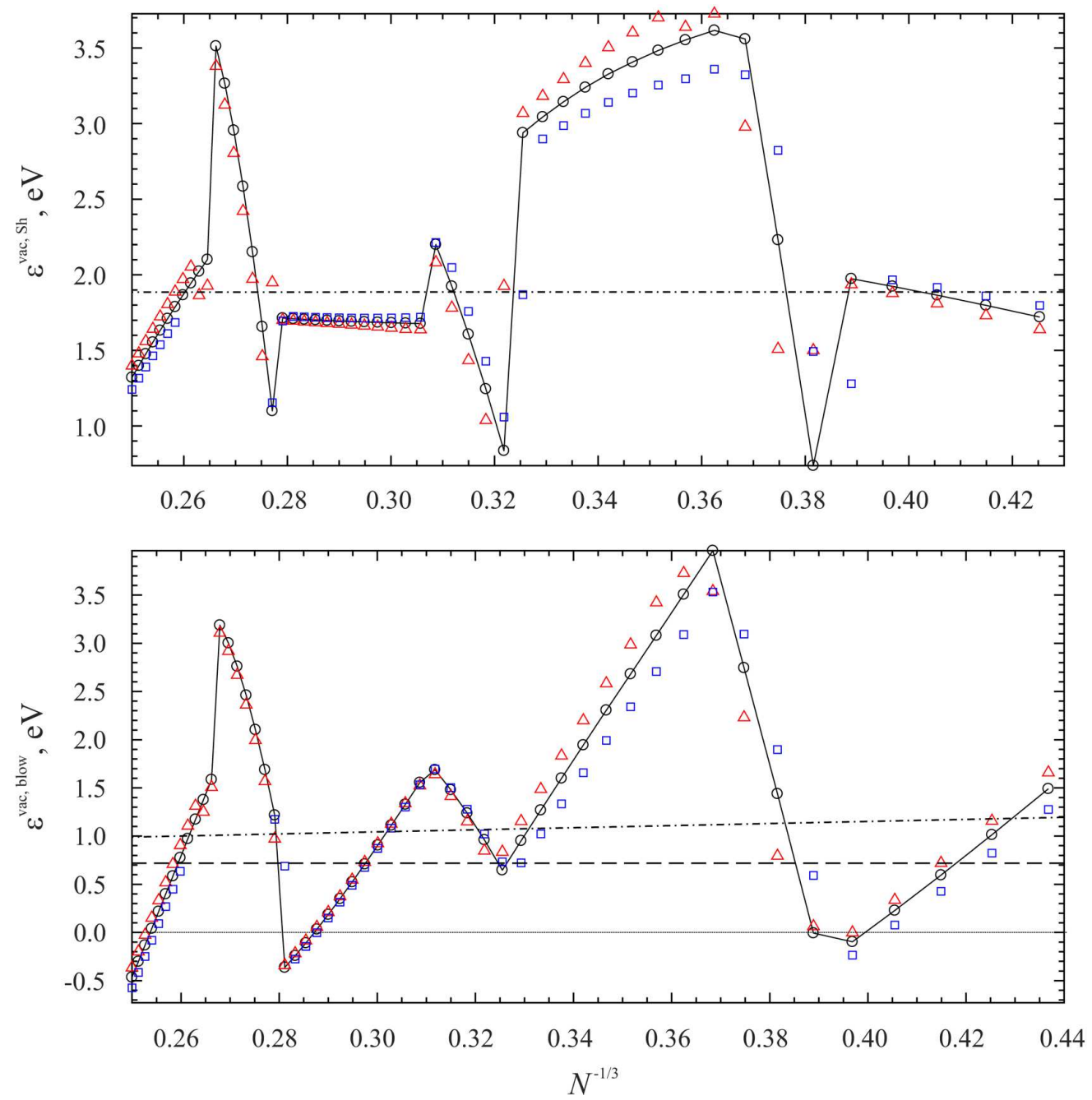

Fig. 8. The same as in Fig. 7, but for Mg. The horizontal dotted line corresponds to $\varepsilon_{\infty}^{\mathrm{vac}}=0.72 \mathrm{eV}$

cially in the case of $\mathrm{Al}$, the values of $\varepsilon_{N, v}^{\mathrm{vac}, \text { blow }}$ become negative.

The difference between the vacancy formation energies in the charged and neutral clusters following both mechanisms $\left(E_{N}^{ \pm} \rightarrow E_{N-1, v}^{ \pm}+E_{\text {at }}\right.$ and $E_{N}^{ \pm} \rightarrow E_{N, v}^{ \pm}$, respectively) can be presented in the form of relations

$$
\begin{aligned}
& \varepsilon_{N, v}^{\mathrm{vac}, \mathrm{Sh},+}=\varepsilon_{N, v}^{\mathrm{vac}, \mathrm{Sh}}+\mathrm{IP}_{N-1, v}-\mathrm{IP}_{N}, \\
& \varepsilon_{N, v}^{\mathrm{vac}, \text { blow },+}=\varepsilon_{N, v}^{\mathrm{vac}, \text { blow }}+\mathrm{IP}_{N, v}-\mathrm{IP}_{N}, \\
& \varepsilon_{N, v}^{\mathrm{vac}, \mathrm{Sh},-}=\varepsilon_{N, v}^{\mathrm{vac}, \mathrm{Sh}}-\mathrm{EA}_{N-1, v}+\mathrm{EA}_{N}, \\
& \varepsilon_{N, v}^{\mathrm{vac}, \text { blow },-}=\varepsilon_{N, v}^{\mathrm{vac}, \text { blow }}-\mathrm{EA}_{N, v}+\mathrm{EA}_{N} .
\end{aligned}
$$

The character of the cluster-size dependence of the vacancy formation energy on the excess charge in the cluster [Eqs. (25)] is completely confirmed by the results of direct calculations (Figs. 7 to 9). This character is determined by the behavior of IP and EA shown in Fig. 2.

The presented results of calculations carried out in the framework of LDA correspond to the zero temperature. It is probable that strong oscillations of energy characteristics will be partially suppressed at atomic concentrations corresponding to finite temperatures, for the lowered symmetry of the cluster shape, and if applying the LSDA for the exchange-correlation energy.

ISSN 2071-0194. Ukr. J. Phys. 2017. Vol. 62, No. 9 

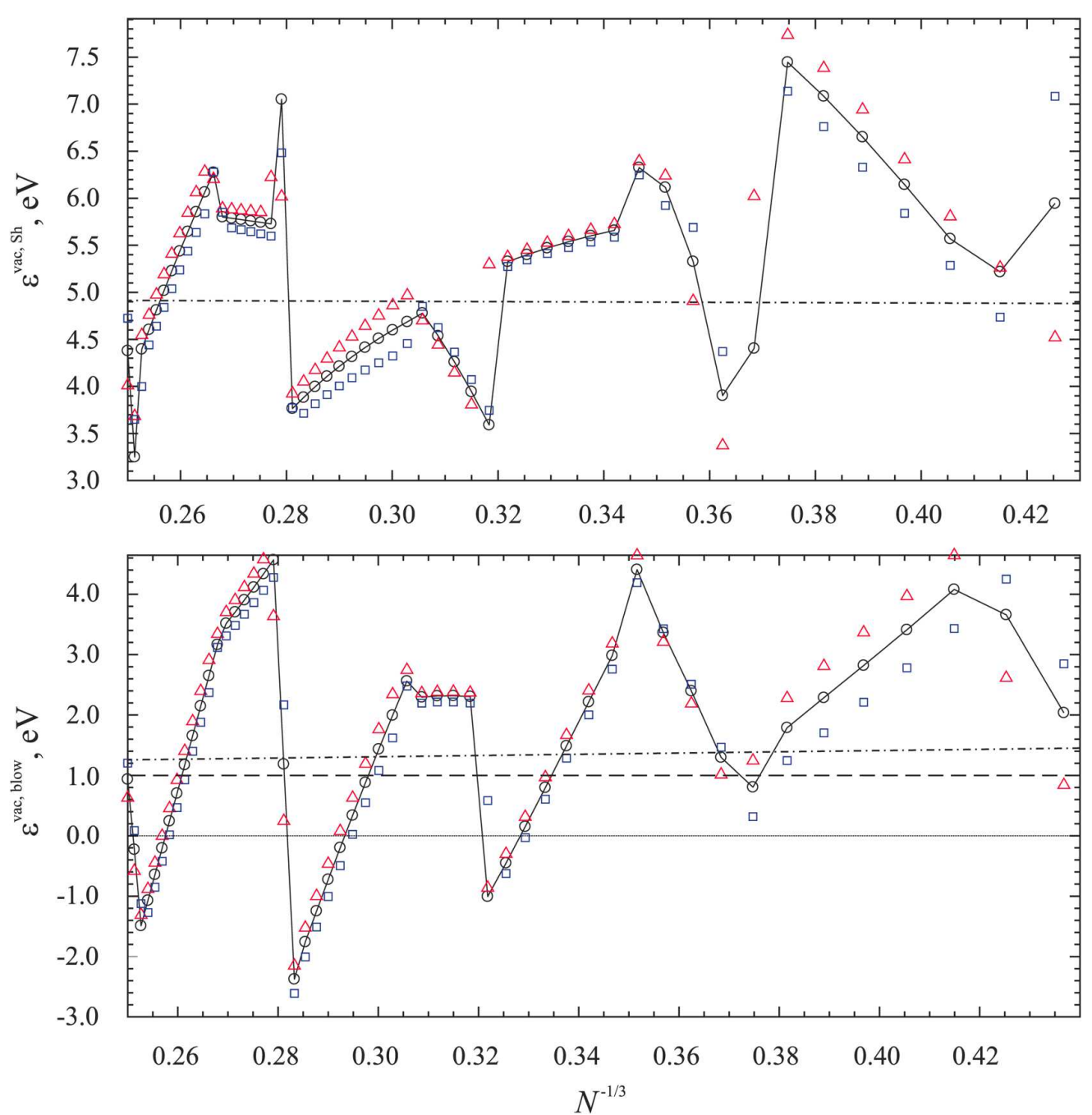

Fig. 9. The same as in Fig. 7, but for Al. The horizontal dotted line corresponds to $\varepsilon_{\infty}^{\mathrm{vac}}=1.0 \mathrm{eV}$

In quasithermodynamics, the expedience for a vacancy to appear in a cluster at a finite temperature $T$ can be estimated from the variation condition for the free energy,

$\Delta F_{N, v}^{\mathrm{vac}, \text { blow }}=\varepsilon_{N, v}^{\mathrm{vac}, \text { blow }}-T \Delta S_{N, v}^{\mathrm{vac}, \text { blow }} \leq 0$.

Since the number of ions in a cluster does not change at the vacancy "blowing", the entropic contribution is only formed by the degenerate electron gas. The corresponding expression looks like

$T \Delta S_{N, v}^{\mathrm{vac}, \text { blow }}=\frac{2 \pi^{5 / 3}}{3^{2 / 3}}\left(\frac{k_{\mathrm{B}} T}{e^{2}}\right)^{2} \times$

ISSN 2071-0194. Ukr. J. Phys. 2017. Vol. 62, No. 9 $\times \int_{0}^{\infty} d r r^{2}\left[n_{N, v}^{1 / 3}(r)-n_{N}^{1 / 3}(r)\right]$.

If formula (27) is applied to calculations, the equilibrium profiles of the electron distribution obtained in the stick-slip jellium model at the given $N$ and $T$ are used. The profiles corresponding to the case $T=0$ and $N=12$ are depicted in Fig. 1.

\section{Conclusions}

Self-consistent calculations of the radial distribution profiles of the electron concentration and the electrostatic potential in continuous (defect-free) metal clus- 
ters and clusters with a vacancy have been performed in the framework of the stabilized jellium model, by using the Kohn-Sham method. As a result, the total energies of a neutral and a charged defect cluster were determined for the first time. The results obtained are used to directly calculate the dissociation, cohesive, and vacancy formation energies, the electron affinity, the ionization potential, and the capacitance. The results of calculations for $\mathrm{Na}, \mathrm{Mg}$, and $\mathrm{Al}$ are compared with the asymptotics and the results obtained for defect-free clusters. The computer code for calculations was created by the authors themselves.

The ionization potential for a small cluster with a vacancy is larger than that for a continuous cluster (approximately by $0.5 \mathrm{eV}$ for $\mathrm{Al}$ and by $0.1 \mathrm{eV}$ for $\mathrm{Na}$ ). The difference is maximum at the transition from the completely filled shell to an empty one. As the number of atoms in the cluster, $N$, increases, this difference vanishes. The magic atomic numbers for continuous clusters and clusters with a vacancy are different, especially in the case of $\mathrm{Al}$ clusters.

The normalized electric capacitances of clusters always exceed unity and contain size-induced quantum fluctuations. For defect clusters with partially filled electron shells, the capacitance is considerably larger than that for continuous ones.

The size dependence of the cohesive energy contains local maxima. The corresponding clusters are more stable; i.e. they have larger values of binding, dissociation, and vacancy formation energies than their neighbors. For small clusters, those maxima mark the termination of the filling of the next electron shell with the growth of the parameter $N$. The positions of the maxima for defect and defect-free clusters are different, which is not only a result of the distinction between their dimensions, but is also associated with the behavior of electron wave functions.

The cohesive energies of anions and cations in charged clusters differ from those in neutral clusters. The positive excess charge gives rise to the energy reduction owing to the growth of electrostatic repulsion forces, whereas the negative excess charge results in the opposite effect.

Quantum-size dependences of the energy of the vacancy formation driven by the Schottky and "bubble blowing" mechanisms, as well as their asymptotic behavior, are determined for the first time. It is shown that the cluster-size asymptotics for those two mechanisms differ from each other, but weakly depend on the number of atoms in the cluster. The character of the size dependence of the vacancy formation energy on the excess charge in a cluster is governed by the behavior of the cluster ionization potential and the electron affinity energy.

In the sections between the maxima, the dissociation energy either increases with the growth of $N$ or has a local minimum. At the same time, the vacancy formation energy monotonically decreases in those sections.

In this model, the relaxation of the cluster volume was not taken into consideration. The influence of the self-compressing on the ionization potential in large clusters was analytically described in works $[20,21]$. For small clusters, this issue was numerically studied in work [16]. The relaxation of the ionic distribution in a cluster gives rise to a reduction in the total energy of a specimen. More consistent, but more complicated, are $a b$ initio methods, in which the coordinates of ions are selected to minimize the total energy of the cluster. This procedure was realized in work [26], but only for $\mathrm{Al}_{30-70}$ clusters, i.e. clusters with a small number of atoms.

The authors express their gratitude to A.V. Korotun for his help in the preparation of the manuscript for the publication.

1. J. Frenkel. Kinetic Theory of Liquids (Dover, 1955).

2. A. Safaei. Cohesive energy and physical properties of nanocrystals. Phil. Mag. 91, 1509 (2011).

3. W. Luo, K. Su, K. Li, G. Liao, N. Hu, M. Jia. Substrate effect on the melting temperature of gold nanoparticles. J. Chem. Phys. 136, 234704 (2012).

4. J. Chandra, K. Kholiya. Diameter-dependent thermodynamic and elastic properties of metallic nanoparticles. Mod. Phys. Lett. B 29, 1550025 (2015).

5. L.A. Bulavin, O.Yu. Aktan, Yu.F. Zabashta. The role of vacancies of a highly deformed crystal in the melting process. Fiz. Tverd. Tela 52, 662 (2010) (in Russian).

6. M.N. Magomedov. On the size dependence of the melting parameters of silicon. Zh. Tekhn. Fiz. 86, 92 (2016) (in Russian).

7. W. Qi. Nanoscopic thermodynamics. Acc. Chem. Res. 49, 1587 (2016).

8. G.A. Breaux, C.M. Neal, B. Cao, M.F. Jarrold. Melting, premelting, and structural transitions in size-selected aluminum clusters with around 55 atoms. Phys. Rev. Lett. 94, 173401 (2005).

9. C. Hock, C. Bartels, S. Straßburg, M. Schmidt, H. Haberland, B. von Issendorff, A. Aguado. Premelting and postmelting in clusters. Phys. Rev. Lett. 102, 043401 (2009). 
10. A.K. Starace, B. Cao, O.H. Judd, I. Bhattacharyya, M.F. Jarrold. Melting of size-selected aluminum nanoclusters with 84-128 atoms. J. Chem. Phys. 132, 034302 (2010).

11. S. Zamith, P. Labastie, F. Chirot, J.-M. L'Hermite. Twostep melting of $\mathrm{Na}_{41}^{+}$. J. Chem. Phys. 133, 154501 (2010).

12. C.C. Yang, S. Li. Investigation of cohesive energy effects on size-dependent physical and chemical properties of nanocrystals. Phys. Rev. B 75, 165413 (2007).

13. S.C. Hendy. A thermodynamic model for the melting of supported metal nanoparticles. Nanotechn. 18, 175703 (2007).

14. G. Guisbiers. Size-dependent materials properties toward a universal equation. Nanoscale Res. Lett. 5, 1132 (2010).

15. W.A. de Heer. The physics of simple metal clusters: Experimental aspects and simple models. Rev. Mod. Phys. 65, 611 (1993).

16. A. Vieira, M.B. Torres, C. Fiolhais, L.C. Balbás. Comparison of the spherically averaged pseudopotential model with the stabilized jellium model. J. Phys. B 30, 3583 (1997).

17. A. Aguado, J.M. Lòpez. Titanium embedded cage structure formation in $\mathrm{Al}_{n} \mathrm{Ti}^{+}$clusters and their interaction with Ar. J. Chem. Phys. 130, 064704 (2009).

18. J.P. Perdew. Simple theories for simple metals: Facedependent surface energies and work functions. Prog. Surf. Sci. 48, 245 (1995).

19. A.V. Babich, V.V. Pogosov. Effects of electron levels broadening and electron temperature in tunnel structures based on metal nanoclusters. Surf. Sci. 640, 210 (2010).

20. I.T. Iakubov, V.V. Pogosov. Towards a theory of selfcompressed metallic clusters. Model of stabilized jellium. Physica A 214, 287 (1995).

21. A. Kiejna, V.V. Pogosov. On the temperature dependence of the ionization potential of self-compressed solid- and liquid-metallic clusters. J. Phys.: Cond. Matter. 8, 4245 (1996).

22. O. Kostko. Photoelectron spectroscopy of mass-selected sodium, coinage metal and divalent metal cluster anions. Ph.D. thesis (University of Freiburg, 2007).

23. A. Halder, V.V. Kresin. Nanocluster ionization energies and work function of aluminum, and their temperature dependence. J. Chem. Phys. 143, 164313 (2015).

24. A.V. Babich, V.V. Pogosov, V.I. Reva. Estimation of the vacancy contribution to the work function of electrons and positrons from metals. Pis'ma Zh. Tekhn. Fiz. 42, No. 20, 11 (2016) (in Russian)

25. V.V. Pogosov. On the tension of curved metal surface. Solid State Commun. 89, 1017 (1994).

26. A.K. Starace, C.M. Neal, B. Cao, M.F. Jarrold, A. Aguado, J.M. López. Electronic effects on melting: Comparison of aluminum cluster anions and cations. J. Chem. Phys. 131, 044307 (2009).

27. V.V. Pogosov. Sum-rules and energy characteristics of small metal particle. Solid State Commun. 75, 469 (1990).
28. M. Seidl, J.P. Perdew, M. Brajczewska, C. Fiolhais. Ionization energy and electron affinity of a metal cluster in the stabilized jellium model: Size effect and charging limit. J. Chem. Phys. 108, 8182 (1998).

29. C. Bréchignac, Ph. Cahuzac, J. Leygnier, J. Weiner. Dynamics of unimolecular dissociation of sodium cluster ions. J. Chem. Phys. 90, 1492 (1989).

30. U. Ray, M.F. Jarrold, J.E. Bower, J.S. Kraus. Photodissociation kinetics of aluminum cluster ions: determination of cluster dissociation energies. J. Chem. Phys. 91, 2912 (1989).

31. P. Ziesche, J.P. Perdew, C. Fiolhais. Spherical voids in the stabilized jellium model: Rigorous theorems and Pade representation of the void-formation energy. Phys. Rev. B 49, 7916 (1994)

32. C. Freysoldt, B. Grabowski, T. Hickel, J. Neugebauer, G. Kresse, A. Janotti, C.G. Van de Walle. First-principles calculations for point defects in solids. Rev. Mod. Phys. 86, 253 (2014).

33. A.V. Babich, P.V. Vakula, V.V. Pogosov. On a vacancy in metal. Fiz. Tverd. Tela 56, 841 (2014) (in Russian).

34. H. Delavari, H.R. Madaah Hosseinia, A. Simchia. Effects of particle size, shape and crystal structure on the formation energy of Schottky vacancies in free-standing metal nanoparticles: A model study. Physica B 406, 3777 (2011).

Received 11.02.17.

Translated from Ukrainian by O.I. Voitenko

В.В. Погосов, В.І.Рева

\section{РОЗМІРНІ ЗАЛЕЖНОСТІ ЕНЕРГЕТИЧНИХ}

ХАРАКТЕРИСТИК ЗАРЯДЖЕНИХ МЕТАЛЕВИХ КЛАСТЕРІВ, ЩО МІСТЯТЬ МОНОВАКАНСІЮ

$\mathrm{P}$ е $з$ ю м е

Методом Кона-Шема у моделі стабільного желе виконано самоузгоджені розрахунки профілів радіальних розподілів електронів та потенціалів суцільних кластерів і кластерів із центрованою моновакансією. Це дозволило вперше одержати повну енергію нейтральних та заряджених дефектних кластерів, на основі чого проведено прямі розрахунки енергій дисоціації, когезіі, утворення вакансії, спорідненості до електрону, потенціалу іонізації, а також електричної ємності. Результати чисельних розрахунків для кластерів $\mathrm{Na}$, $\mathrm{Mg}$ i Al порівнюються з асимптотиками і результатами для бездефектних кластерів. Розраховано квантово-розмірні залежності енергії утворення вакансії за механізмами Шотткі i "видування пухирця" і визначено їх асимптотичну поведінку. Виявлено сильні розмірні флуктуації енергії утворення вакансії в усій області розмірів кластерів. Показано, що розмірні асимптотики цих двох механізмів відрізняються одна від одної і слабко залежать від числа атомів у кластері. Обчислення проводилися на кластері CKIT-3 Інституту кібернетики ім. В.М. Глушкова НАН України $\left(R_{\text {peak }}=7,4 T_{\text {flops }}\right)$. 